\title{
Entre théorie des passions et rhétorique de la conversation : Cureau de la Chambre et « Les Charactères du Ris » (1640)
}

Bernard Roukhomovsky

\section{(2) OpenEdition}

\section{Journals}

Édition électronique

URL : http://journals.openedition.org/recherchestravaux/257

DOI : 10.4000/recherchestravaux.257

ISSN : 1969-6434

Éditeur

UGA Éditions/Université Grenoble Alpes

Édition imprimée

Date de publication : 30 mai 2005

Pagination : 25-39

ISBN : 0151-1874

ISSN : 0151-1874

Référence électronique

Bernard Roukhomovsky, « Entre théorie des passions et rhétorique de la conversation : Cureau de la Chambre et «Les Charactères du Ris » (1640) », Recherches \& Travaux [En ligne], 67 | 2005, mis en ligne le 30 septembre 2008, consulté le 08 septembre 2020. URL : http://journals.openedition.org/ recherchestravaux/257; DOI : https://doi.org/10.4000/recherchestravaux.257 
Bernard ROUKHOMOVSKY

Université Stendhal - Grenoble 3

\title{
Entre théorie des passions et rhétorique de la conversation: \\ Cureau de la Chambre et \\ «Les Charactères du Ris» (I640)
}

\begin{abstract}
Monsieur, on peut se fier à mes observations. Tenez, je n'ai qu'à regarder une femme entre deux yeux, je vous dirai ce qu'elle sent et ce qu'elle sentira, le tout à une virgule près. Tout ce qui se passe dans son cœur s'écrit sur son visage, et j'ai tant étudié cette écriture-là, que je la lis tout aussi couramment que la mienne.
\end{abstract}

MarivauX, La Fausse Suivante ou Le Fourbe puni, III, sc. 3

C'est par des biais différents que la théorie des passions d'une part, la théorie de la conversation de l'autre, abordent au XVII siècle le délicat sujet du rire. Les questions posées par la première (De quel mouvement passionnel le rire est-il le signe, et pourquoi ?) ne sont pas exactement celles formulées par la seconde (Est-il permis de rire dans la conversation, dans quelles limites et de quoi ?). Il reste que les deux approches - qui représentent deux veines majeures de la littérature morale du Grand Siècle - sont étroitement intriquées: c'est ainsi que, dans Les Passions de l'âme (I649), Descartes passe de la première - la description psychophysiologique du Ris (articles CXXIV-CXXVII) - à la seconde - les articles CLXXX-CLXXXI règlent l'"usage de la Raillerie» et fixent les conditions d'un bon usage $\mathrm{du}$ "Ris en la raillerie ${ }^{\mathrm{I}}$ ». Le discours sur les passions rencontre nécessairement la question (rhétorique s'il en est!) des passions dans le discours. Aussi, il est permis de penser que la question de son usage (bon ou mauvais) dans la conversation constitue presque toujours un enjeu latent de la réflexion sur le rire à l'âge classique, quand bien même

I. Descartes, Les Passions de l'âme, éd. G. Rodis-Lewis, Paris, Vrin, I991, p. I53-156 et 196197. 
celle-ci se donne pour objet principal de rendre compte du mouvement passionnel dont il est le signe et de la mécanique corporelle dont il est l'effet. On voudrait proposer ici de relire dans cette perspective le long chapitre que Marin Cureau de la Chambre (I596-I669) consacre, dans ses Charactères des Passions (I640), aux «Charactères du Ris ${ }^{2} »$.

\section{Quand rire, c'est dire}

Le succès rencontré par Les Charactères des Passions (on dénombre six éditions jusqu'en 1662), venant notamment après celui du Tableau des Passions humaines de Nicolas Coëffeteau (sept éditions se suivent de I620 à I632) 3, illustre la fortune persistante d'un genre - la théorie des passions ${ }^{4}$ - que Cureau, se prévalant de sa formation et de sa "profession", a l'ambition de renouveler en l'abordant en médecin, en posant les jalons d'une «anthropologie médicales»:

Ce n'est pourtant pas que je croie être le premier qui ait pris garde à ce qui manquait à l'entière connaissance des Passions. [...] Mais comme ce sont des Actions communes à l'Âme et au Corps, et qu'il faut que la Médecine et la Philosophie Morale se secourent l'une l'autre pour en parler bien exactement, il est arrivé que ceux qui l'ont voulu entreprendre, ne les y ont pu employer toutes deux $[\ldots]{ }^{6}$

Linfluence de la tradition médicale - à commencer par celle, probable, de Laurent Joubert et de son Traité du Ris (I579) - n'est sans doute pas étrangère à la place particulière que Cureau choisit de faire au rire. Contrairement à Coëffeteau, qui fait entrer la description du Ris dans le chapitre «Des effets de la Volupté» et tient qu'il «n'est pas une Passion, mais un effet extérieur de la Passion intérieure7", le médecin conçoit le rire comme passion à part entière, à côté (et à la suite) de la joie - tout comme le fera Le Brun dans sa

2. M. Cureau de la Chambre, Les Charactères des Passions, Paris, P. Rocolet, I640, chap. IV. Nos références vont au texte de la 6 éd. (Paris, J. d'Allin, I662); nous en avons modernisé l'orthographe.

3. N. Coëffeteau, Tableau des Passions humaines, de leurs causes et de leurs effets, Paris, S. Cramoisy, I620.

4. Sur l'histoire de la théorie des passions en France dans la première moitié du XVIIe siècle et sur la place qu'y tient Cureau, voir, outre l'ouvrage classique de A. Levi, French Moralists. The Theory of the Passions, I585 to 1649, Oxford, Clarendon Press, 1964, W. Riese, La Théorie des passions à la lumière de la pensée médicale du XVII siècle, Bâle et New York, Karger, 1965, et surtout l'ouvrage récent de L. Desjardins, Le Corps parlant. Savoirs et représentations des passions au XVII' siècle, Québec, Presses de l'université Laval et Paris, L'Harmattan, 20or ; signalons enfin les belles pages de M. Escola sur «Marin Cureau de la Chambre: l'ordre du sémiotique (1640-1659)» dans sa thèse de doctorat, Rhétorique du discontinu, université Paris 4, 1995), vol. I, p. 175-199.

5. Selon la formule de W. Riese, (La Théorie des passions..., op. cit., p. 19). Rappelons que Cureau, médecin au Mans en I630-I63I, acheta en I65o la charge de médecin ordinaire du roi.

6. M. Cureau de la Chambre, Les Charactères des Passions, op. cit., "Avis nécessaire au lecteur", n. p.

7. N. Coëffeteau, Tableau des passions humaines..., op. cit., p. 282. 
typologie des expressions passionnelles ${ }^{8}$. C'est un parti pris théorique assez original - au regard des divisions de l'École - pour qu'il juge bon de s'en expliquer dès l'avis liminaire9 et pour qu'il le justifie de nouveau dans le chapitre III («Les Caractères de la Joie»), où il mentionne le Ris (comme effet corporel) au nombre des "caractères que la joie imprime sur le corps par le commandement de l'âme», avec les "Regards riants ", la "Sérénité du front", les «Caresses» et l' «Inquiétude» (l'Impatience):

Pour le Ris, quoiqu'il semble être un effet particulier de la Joie, il ne se rencontre pas toujours avec elle. Et quand il l'accompagne, ce n'est pas à elle seule qu'il doit sa naissance; il y a d'autres causes qui y contribuent et qui excitent dans l'âme une émotion différente de celle du plaisir : c'est pourquoi nous n’avons pas craint de l'appeler une passion, ne considérant pas seulement le mouvement extérieur qui parait sur le visage, mais celui que l'âme souffre au dedans, dont nous examinerons la nature et les effets au chapitre suivant. ${ }^{\text {Io }}$

Cette innovation ne va pas sans équivoque - puisque le même mot en vient à désigner sous la plume du médecin le mouvement corporel appelé rire et le mouvement passionnel dont il est la marque (le "caractère»). Ce n'est là qu'un expédient taxinomique (dont Cureau prend soin de minimiser l'audace et l'impact) ${ }^{\mathrm{II}}$ destiné à préserver, malgré l'apparente diversité des causes du rire $^{\mathrm{I} 2}$, le caractère biunivoque du rapport entre l'ordre des passions et celui de leurs signes, qui fonde l'intelligibilité - à proprement parler la lisibilité - des expressions passionnelles:

[...] je ne puis m'imaginer que la Nature qui est si régulière et si uniforme en toutes ses autres actions, se soit oubliée en celle-ci; qu'elle ait voulu donner diverses causes à un même effet; et qu'étant véritable que toute sorte de Ris a quelque chose de commun, l'Âme n'ait pas un motif général d'une action commune et générale. ${ }^{13}$

8. Ch. Le Brun, Conférence sur l'expression des passions, reproduite dans Nouvelle Revue de Psychanalyse, $\mathrm{n}^{\circ}$ 2I, 1980, p. 93-I2I.

9. M. Cureau de la Chambre, Les Charactères des Passions, op. cit.: "Car je ne considère pas le Ris comme un pur effet corporel; mais j'y comprends l'émotion de l'âme qui le cause, et en cette considération il peut passer pour une passion particulière [...].»

Io. Ibid., chap. III, p. I84.

II. Ibid., "Avis nécessaire au Lecteur» : "Ne t'arrête pourtant pas à cela, il est indifférent que c'en soit une [passion], ou que ce n'en soit que l'effet: il y a beaucoup de choses que je n'examine pas ici avec la sévérité de l'École.»

I2. Ibid., p. 198: "Car on voit que le Ris vient des actions et des paroles plaisantes et facétieuses, de l'admiration, du dépit, du mépris, des caresses, du chatouillement et de quelques maladies: et comme d'abord il semble qu'il n'y ait aucun rapport entre toutes ces choses, on peut facilement croire que le Ris est un mot équivoque qui marque des effets de différente nature $[\ldots] . »$

13. Ibid., p. 206. Le même problème se posera dans le cas des larmes et trouvera la même solution: "Car bien qu'elles n'aient été prises jusqu'ici que pour un effet et un Caractère de la 
Ce qui est en jeu, de fait, c'est la possibilité de donner un sens au rire ou, ce qui revient au même, de résorber l'énigme du rire dans le cadre d'une herméneutique et d'une sémiologie des expressions passionnelles. Or, il ne s'agit pas seulement de s'entendre sur ce que rire veut dire, il s'agit aussi de s'assurer qu'en toute circonstance, rire, c'est dire. En quoi Cureau rejoint le consensus général selon lequel le rire est investi d'une fonction sémiotique: pour Cramail, c'est «un branlement ou une vibration, tant du diaphragme que des muscles de la bouche, par laquelle l'homme signifie et découvre extérieurement deux mouvements, ou passions de l'âme, à savoir l'admiration et la joie ${ }^{\mathrm{I} 4}$ »; selon Furetière, il consiste à "donner des témoignages d'une joie intérieure par des signes extérieurs, soit par l'éclat de la voix, soit par les mouvements du visage ${ }^{15}$.» Mais cette application à penser le rire comme signe est particulièrement marquée chez Cureau, qui se propose de montrer qu'en toute circonstance rire veut dire quelque chose, le rire même provoqué par le simple chatouillement n'échappant pas à la règle:

De sorte que le Ris qui accompagne ce mouvement est un témoignage que l'âme veut rendre, du plaisir qu'elle reçoit, et que la personne qui l'excite lui est agréable. ${ }^{16}$

Ayant ainsi provisoirement repoussé le problème de l'automatisme physique - sur lequel nous aurons pourtant à revenir -, Cureau pose en règle générale que le rire est doublement signifiant, puisque non seulement fait signe en lui le mouvement passionnel qui s’y donne à lire, mais encore la volonté de l'âme de le donner à lire, de dessein délibéré:

Voilà quelle est la nature de cette passion, d'où à mon avis il est aisé de tirer le motif des objets ridicules. Car bien qu'il semble que nous soyons dans le même sentiment de ceux qui ont mis le ridicule dans les choses nouvelles et agréables [...], néanmoins, si l'on prend garde à ce que nous avons dit, on verra qu'il y a une grande différence; parce que nous ajoutons à la nouveauté une circonstance qu'ils n'y mettent point, savoir est que l'âme veut témoigner la surprise que cette nouveauté lui donne. ${ }^{17}$

De là à conclure que le rire est "principalement destiné pour la conversation» (dans le sens compréhensif que ce terme revêt alors), qu’il n’a de sens et

Douleur, elles prétendent néanmoins que l'âme leur ayant affecté un mouvement particulier et un motif différent de celui de la Tristesse, elles méritent d'entrer dans l'ordre et dans la famille des Passions [...].» («Les Caractères des Larmes»)

I4. Discours académique du Ris prononcé en l'Académie des Philarèthes, dans Les Jeux de l'inconnu, Paris, I630, p. 7 (je souligne).

I5. Je souligne.

I6. M. Cureau de la Chambre, Les Charactères des Passions, op. cit., chap. IV, p. I98-I99 (je souligne).

17. Ibid., p. 199 (je souligne). 
n'est d'usage qu'en situation d'interaction sociale, il y a un pas que le médecin s'empresse de franchir:

Mais ce que j'estime de plus considérable pour connaître la nature du Ris, est qu'il se fait rarement quand on est seul, et que la plupart des objets qui l'excitent puissamment dans la conversation, ne l'émeuvent pas du tout dans la solitude. De sorte qu'il est vraisemblable que la compagnie sert de quelque chose à sa production, et que l'âme veut faire voir qu' elle est surprise; ce qui serait inutile s'il n'y avait quelqu'un qui fût témoin de ce qu' elle veut faire. C'est pourquoi elle ne doit point exciter le Ris quand on est seul. ${ }^{8}$

Aussi, sur ce rapport organique entre rire et conversation, Cureau revient à plusieurs reprises:

C'est pourquoi il y a des choses nouvelles et agréables qui ne font pas rire, d'autant que l'âme ne prétend pas de faire paraître le sentiment qu'elle en a: ainsi quand on est seul et que l'on pense à quelque chose de plaisant, on n'en rit pas d'ordinaire; mais seulement quand on en fait le récit: parce qu'alors l'âme fait dessein de témoigner la surprise qu'il lui a causée. ${ }^{19}$

Et s'il est vrai "que l'on rit souvent quand on est seul», concède Cureau, «cela arrive par l'erreur de l'imagination qui se détourne de la fin que la nature lui a prescrite», de la même façon que, si l’on voit des gens qui "parlent tout seuls», c'est "contre l'intention de la nature qui a destiné la voix et la parole pour être des instruments de la société, et pour servir à la communication que les animaux doivent avoir ensemble ${ }^{20}$ ». On mesure ici le soin que met l'auteur à assigner au rire un rang parmi les "instruments de la société», au plus près du langage articulé (au plus près de la parole et de la voix), au point de rencontre entre la théorie des passions et la civilité classique, la fonction sémiotique qui lui est dévolue par la première le qualifiant comme instrument de la seconde (en quoi il y a continuité de l'une à l'autre). Mieux encore, par affinités électives, il semble que le rire ait doublement partie liée avec la sociabilité, puisque non seulement c'est un moyen de la conversation (en dehors de laquelle il n'y a pas lieu de rire), mais encore c'est dans la conversation (ou dans ses dérèglements) que l'on trouve le plus facilement matière à rire (le rire se comprenant alors comme sanction des manquements à l'esprit de la conversation):

[...] le Ris étant principalement destiné pour la conversation, les objets qui la regardent particulièrement sont aussi ceux qui font plus facilement rire. Telles sont les actions et les paroles facétieuses qui comprennent tout ce qui est difforme et malséant, les dommages légers faits à dessein ou reçus par sottise, les tromperies de petite conséquence, les railleries, en un mot toutes les difformités sans

18. Ibid., p. 192-193.

19. Ibid., p. 199-200.

2o. Ibid., p. 200. 
douleur ${ }^{21}$ : car toutes ces choses excitent le Ris, parce qu'elles marquent le défaut des qualités nécessaires à la conversation, comme de la bonne grâce, de la bienséance, de l'accortise, de la bonté, et des autres; l'esprit se trouve surpris quand il voit des actions contraires à ces vertus qui sont les fondements de la société et de la vie civile. ${ }^{22}$

\section{Une «marque naturelle»}

Il apparaît que la question du rire, telle qu'elle est posée et traitée par Cureau, est coextensive à une réflexion sur les signes, sur ce qui permet leur déchiffrement (la sémiologie des expressions passionnelles par exemple) et sur ce qui régule leur circulation (l'esthétique de la conversation). Cette réflexion, qui aboutit à l'émergence d'une "anthropologie sémiotique ${ }^{23}$ ", est la même qui sous-tend l'entreprise de Cureau et qui, tout aussi bien, fait dire à Descartes quelques années plus tôt, au début de son traité du Monde (I632-I635), que la Nature «a établi les ris et les larmes pour nous faire lire la joie et la tristesse sur le visage des hommes 24 ». Pour autant, même si le problème posé, chez l'un comme chez l'autre, est bien celui de la lisibilité des expressions passionnelles, ce qui n'est bientôt plus chez Descartes (dont on connaît la défiance à l'égard des évidences sensibles) qu'une simple image en passant, demeure imprégné, chez Cureau, d'une valeur très concrète qui en condense la signification profonde, et la précise.

C'est en effet dans un sens très concret que s'entend sous sa plume le terme clé de caractère, constamment associé à un réseau lexical à forte connotation typographique («marque», «marquer», «imprimer»...)25: que le ris soit rangé parmi les "Caractères que la Joie imprime sur le corps» ou qu’il soit considéré comme une passion dont il importe, pour en "connaître la nature», de "marquer les Caractères", dans les deux occurrences "Caractère» se comprend, si l'on ose dire, à la lettre. La question du rire est ainsi prise dans le projet de

2I. La référence (canonique) à la définition aristotélicienne du ridicule se justifie d'autant mieux qu'il n'y a pas solution de continuité, pour les théoriciens de l'époque, entre l'esthétique théâtrale et celle de la conversation. Voir à ce sujet É. Bury, Littérature et politesse. L'invention de l'honnête homme (I580-1750), Paris, Presses universitaires de France, I996, chap. III.

22. Ibid., p. 195 (je souligne).

23. J'emprunte la formule à H. R. Jauss ("Vom plurale tantum der Charaktere zum singulare tantum des Individuums ", in Individualität, Munich, W. Fink, 1988, p. 237-269).

24. Le Monde ou Traité de la lumière, dans Euvres de Descartes, éd. Adam-Tannery, Paris, Cerf, i897-1909, t. VI, p. 4 (je souligne).

25. Sur les métaphores typographiques associées au terme et à la notion de caractère, et sur le profit que l'on retire à «ne pas perdre de vue la valeur originelle, très concrète et matérielle, de telles métaphores", voir L. Van Delft, Littérature et anthropologie. Le caractère à l'âge classique, Paris, Presses universitaires de France, 1993, p. 19-20. 
constituer un alphabet des passions, c'est-à-dire une typologie des signes au moyen desquels la passion se rend lisible et s'invite dans la conversation ${ }^{26}$. Cet alphabet comprend à la fois des "Caractères moraux" (dans la mesure où les passions de l'âme se donnent à lire au travers des conduites) et des "Caractères corporels", qui consistent dans les altérations que le mouvement passionnel fait subir au corps, et singulièrement à la forme du visage - à cet "Air fixe et naturel, où les parties ne se meuvent point et qui n'est pas un effet des émotions de l'âme ${ }^{27}$ » (surface neutre et table d'écriture dont le rôle est comparable à celui de la Tranquillité dans la typologie de Le Brun). Et c'est en vue de prendre toute la mesure de ces altérations que le médecin choisit de privilégier, au détriment de sa forme atténuée (le "Souris») ou moyenne (le «Ris médiocre»), la description d'une forme accentuée du rire (le «Ris véhément»):

Or, comme il peut être faible, médiocre, ou véhément, il est certain que c'est principalement de ce dernier dont il faut marquer les Caractères, parce qu'en tout genre de choses le plus grand doit toujours être la mesure du plus petit; et parce que ses effets sont plus sensibles que ceux des autres: voire même l'on peut dire qu'il n'y a point de passions, quelque violentes qu'elles soient, qui causent de si grands changements au corps que fait celle-ci. ${ }^{28}$

On entrevoit dès à présent les conséquences paradoxales de ce parti pris, puisque le "Ris véhément» est à la fois la forme la plus marquée de la "Passion du Ris» - et, partant, la plus éloquente (ou la plus "parlante») - et la moins licite dans la conversation, eu égard aux exigences de mesure qui régissent celle-ci en contexte classique. On y reviendra, mais il importe auparavant de rendre compte de l'effort entrepris par Cureau pour (sub)ordonner à un processus sémiotique le processus somatique impliqué dans le rire (en tant que mouvement corporel associé à la "Passion du Ris ${ }^{29}$ »). Particulièrement éclairantes, sous ce rapport, sont les précisions données dans l'avis liminaire sur la nature des «Caractères corporels»:

C'est donc une chose certaine, que le corps s'altère et se change quand l'âme s'émeut, et que celle-ci ne fait presque point d'actions qu'elle lui en imprime les marques, que l'on peut appeler Caractères, puisquills en sont les effets, et quills en portent limage et la figure. $3^{30}$

26. On voit ici s'esquisser le vaste projet dont Les Caractères des Passions ne constituent que le premier volet: dans son Art de connaître les hommes (I659-I666), où il revisitera, avant Le Brun, la tradition physiognomonique, l'auteur poursuivra l'étude des «Caractères visibles et manifestes qui paraissent sur le visage» (Première partie, Paris, Rocolet, 1659, p. 2).

27. M. Cureau de la Chambre, Les Charactères des Passions, op. cit., p. 6.

28. Ibid., chap. IV, p. I78-I79 (je souligne).

29. Pour sortir de l'équivoque induite par l'option taxinomique de Cureau, on réservera désormais le terme de "rire» à ce mouvement corporel.

30. M. Cureau de la Chambre, Les Charactères des Passions, op. cit., p. I8 (je souligne). 
S’il est entendu que les mouvements corporels constituent les signes (les «marques») des mouvements de l'âme auxquels ils sont associés, la question se pose de savoir comment se noue le rapport entre les passions et leurs signes. Or, sont ici combinées, peut-être momentanément confondues, deux façons de faire signe, en lesquelles on reconnaît respectivement (selon la terminologie de Peirce remise à l'honneur par $\mathrm{M}$. Escola) ${ }^{3 \mathrm{I}}$ le régime indiciel et le régime iconique du signe (les mouvements corporels sont les signes des passions pour autant "qu'ils en sont les effets» et pour autant "qu'ils en portent l'image et la figure»). Sans préjuger de la tension, qu'il révélera pour finir et sur laquelle nous aurons à revenir, entre ces deux modalités, le chapitre sur le rire illustre néanmoins la prégnance du modèle iconique, et s'attache exemplairement à rapporter la nature des "Caractères corporels" à la nécessité de "trouver dans les choses sensibles une sorte de mouvement qui puisse représenter l'agitation que l'âme souffre ${ }^{32}$ » et, partant, la rendre visible, c'est-à-dire immédiatement lisible: si «le mouvement extérieur qui paraît sur le visage» est l'effet de "celui que l'âme souffre au-dedans", ce rapport de cause à effet est en quelque sorte ordonné et subordonné à un rapport de représentation, donc de similarité. Et c'est bien cette relation de ressemblance entre les deux mouvements que le chapitre sur le rire vise tout d'abord - et ne réussit pas complètement pour finir - à mettre en évidence.

Ainsi, une fois rappelées les conditions typiques dans lesquelles le rire a vocation à se produire (I. «dans la conversation », 2. confrontée à quelque surprise agréable, 3. «l'âme veut faire voir qu'elle est surprise»), Cureau poursuit en ces termes:

Il ne faut pas pourtant croire qu'en ce dessein elle se serve du Ris comme d'une marque prise à plaisir, telles que celles qui partent de notre choix et de notre invention: mais comme d'une marque naturelle qui a une connexion nécessaire avec l'émotion qu'elle représente.

Pour savoir quelle est cette connexion et la raison particulière qui oblige l'âme à se servir de ce mouvement plutôt que d'un autre pour marquer la surprise où elle est, il faut supposer qu'en toute surprise l'âme se retire et rentre en elle-même, la rencontre d'une chose imprévue s'opposant à la liberté de ses pensées, et la contraignant de se recueillir pour mieux discerner l'objet qui se présente. Et alors si elle a dessein de faire paraître en quel état elle est, il faut par la loi qui proportionne les organes et les effets à leurs causes, qu'elle excite quelque mouvement

3I. Voir note 20. En démontrant que, chez Cureau, «le fondement iconique du signe laisse progressivement la place à une structure indicielle», en reflet "de la mutation du statut du signe au début du XVII ${ }^{e}$ siècle» (op. cit., p. 199), M. Escola confirme et développe les hypothèses que j'avais présentées à l'occasion d'une conférence sur «Le rire et le corps dans la théorie des passions (I620-I668)", donnée le II décembre 1993 dans le cadre du Groupe d'étude des moralistes (Paris 4), et dont le présent travail est partiellement inspiré.

32. M. Cureau de la Chambre, Les Charactères des Passions, op. cit., p. I66 (je souligne). 
dans les parties extérieures qui soit semblable à celui qu'elle souffre; et par conséquent qu' elle fasse retirer les muscles vers leur origine, comme elle se retire et se ramasse en elle-même.33

Le rire est donc explicitement défini comme signe naturel (il est ici question d'une "marque naturelle», un peu plus loin d'un «langage naturel ${ }^{34 »)}$ : au rebours d'un signe d'institution, il est lié à la passion qu'il représente par une "connexion nécessaire». Celle-ci consiste dans un rapport de ressemblance, tant et si bien que le rire donne à voir, comme par transparence et sur un mode essentiellement iconique à ce stade, la passion dont il est le signe (on aura compris qu'il s'agit selon Cureau de "quelque surprise agréable»). S’il en est le signe, c'est pour autant qu'il en forme l'image (le mouvement des muscles se fait à l'image du mouvement de l'âme), et c'est là du reste ce qui le qualifie pour la conversation - conversation pour l'oeil plus encore que pour l'oreille - si l'on en croit les premières lignes de l'ouvrage:

La Nature ayant destiné l'homme pour la vie Civile ne s'est pas contentée de lui avoir donné la langue pour découvrir ses intentions; elle a encore voulu imprimer sur son front et dans ses yeux les Images de ses pensées; afin que s'il arrivait que sa parole vînt à démentir son cœur, son visage pût démentir sa parole. ${ }^{35}$

Non seulement il faut convenir ici «que Cureau n'a pas atteint à la pureté du cartésianisme qui conteste à l'âme espace et étendue ${ }^{36}$ », mais encore il faut remarquer que son explication du rire, jusqu'à ce point du moins, se rattache davantage à l'univers de pensée propre au Tableau des Passions humaines - et, partant, à l'épistémè du début du siècle - qu'à celui des Passions de l'âme. Il suffit, pour s'en convaincre, de comparer le texte de Cureau (ce que nous en avons lu jusqu’à présent) avec la description du Ris donnée par Coëffeteau dans le chapitre "Des effets de la Volupté»:

Il n'y a premièrement personne qui ne s'aperçoive qu'au milieu de la joie que nous recevons notre cœur vient à s'épanouir, et comme à s'ouvrir d'aise, dont il envoie les marques et les signes au visage par le Ris qu'il excite à la bouche, où il apporte un visible changement. [...]

De cette joie qui fait épanouir le cour comme une fleur, naît le ris [...]. Car la douceur du plaisir fait que le cour se meut et souvre pour en recevoir l'image: ni plus ni moins que nous allons au devant, et tendons les bras à notre ami, quand il se présente à nous. Et ce sien mouvement, cette aise intérieure, parvient jusqu'au visage, mais paraît principalement à l'ouverture de la bouche, où le ris se forme, et où il a son siège, et de là s'épand aux yeux et au reste de la face [...].37

33. Ibid., p. 193-194 (je souligne).

34. Ibid., p. 197.

35. Ibid., p. I (je souligne).

36. W. Riese, La Théorie des passions..., op. cit., p. 26.

37. N. Coëffeteau, Tableau des Passions humaines..., op. cit., p. 282 (je souligne). 
Si l'explication se double un peu plus loin d'une description psychophysiologique probablement inspirée de Vivès ${ }^{38}$, on voit bien que, comme tentera de le faire encore, quelque vingt ans plus tard, l'auteur des Caractères des Passions, elle (sub)ordonne un ordre somatique à un ordre sémiotique et (pré)suppose, entre le rire et la passion qu'il donne à lire, une relation d'isomorphisme, une similitude immédiatement sensible: le processus de ce rire en forme de fleur (le poète ne dit-il pas que "la rose rit au soleil»?) est celui d'un épanouissement propagé etlou répété, de l'âme au cœur, du cœur à la bouche et de la bouche "aux yeux et au reste du visage»; à l'éclosion du cœur répond exactement celle de la bouche et du visage, qui en forme l'image, c'està-dire le signe.

On objectera que, prenant le contre-pied de cette rêverie de l'éclosion, Cureau, tout au contraire, fait voir dans le rire un mouvement systolique, la contraction des muscles du visage répondant au mouvement de l'âme qui «se retire et se ramasse en elle-même» et, partant, le donnant à lire. Mais cela tient au fait que Cureau ne s'accorde pas avec l'auteur du Tableau des Passions humaines pour rapporter le rire aux effets de la Joie (aussi bien Cureau luimême, dans le chapitre qu'il leur consacre par ailleurs, associe-t-il les effets de la Joie au tropisme de la dilatation), et qu'à l'inverse, fidèle à une double tradition rhétorique (Quintilien) et médicale (Fracastor), il fait entrer la Surprise pour une part essentielle dans cette passion composite qu'il choisit d'appeler la «Passion du Ris». Pour autant, les deux analyses sont également informées par le projet de comprendre le rire comme image sensible, comme figure du mouvement passionnel (quel qu'il soit) dont il est par ailleurs l'effet, d'ordonner le rapport de causalité à un rapport d'affinité, sinon de spécularité: en d'autres termes, elles reposent, semble-t-il, sur le même soubassement épistémologique.

Est-ce à dire que, "au seuil de l'âge classique», le signe ne "cesse" pas encore - en tout cas pas si nettement, si complètement que le voulait Foucault - «d'être lié à ce qu'il marque par les liens solidaires et secrets de la ressemblance 39 »? En vérité, si la théorie du rire développée par Cureau demeure partiellement - et même assez largement - tributaire d'une épistémè préclassique (selon la partition foucaldienne), il reste que les mutations majeures

38. Le ris, poursuit en effet l'auteur, «vient d'une prompte et soudaine émotion de notre âme, qui voulant exprimer la joie, excite une grande abondance de sang chaud, et multiplie les esprits vitaux, qui agitent et remuent les muscles qui sont à l'entour du cœur, et ceux-là agitent et font soulever ceux qui sont attachés aux deux côtés de la bouche, qui pour cette occasion s'ouvre avec un visible changement de toute la forme du visage» (ibid., p. 286).

39. M. Foucault, Les Mots et les choses. Une archéologie des sciences humaines, Paris, Gallimard, I966 (Bibliothèque des sciences humaines), p. 72. 
que, dans le champ de la théorie des passions, le traité cartésien viendra parachever neuf ans plus tard, ne sont pas néanmoins sans l'affecter, ni sans en affecter la cohérence.

\section{Contrepoint mécaniste}

Pour peu, en effet, que l'on entre plus avant dans la lecture des «Caractères du Ris ", on s'avise que le texte n'en est pas homogène. On observe notamment que la «logique de la ressemblance» qui en informe la démarche entre en concurrence avec une conception mécaniste plus ou moins balbutiante, et fait remarquable - que cette logique échoue à rendre raison de tous les effets du Ris (de tous les "Caractères» de la «Passion du Ris»):

Léclat des yeux, la rougeur du visage, et les larmes viennent principalement de la Joie: tous les autres viennent de la Surprise qui fait retirer les muscles vers leur origine; l'âme se servant de ce mouvement extérieur pour faire voir celui qu'elle souffre intérieurement, parce qu'elle se retire en elle-même quand elle est surprise, comme nous avons dit. De sorte que cette contraction des muscles est comme le principe de tous les autres effets du Ris; et peut-être n'y a-t-il qu'elle qui se fasse par le commandement de l'âme, tout le reste se faisant par nécessité et sans dessein. Car il n'est pas vraisemblable que l'ame ait intention de faire ces plis et ces rides qui se voient au coin des yeux, de tenir les yeux à demi fermés et la bouche ouverte, de rendre la voix éclatante et entrecoupée, et ainsi des autres: mais ce sont des effets qui par une suite nécessaire accompagnent le mouvement des muscles. 40

On voit que l'auteur procède ici à une partition décisive, en reflet des tensions qui travaillent son discours et qui en fissurent le socle. D'un côté, donc, le "principe de tous les autres effets du Ris", à savoir la "contraction des muscles»: s'il est seul imputable à une intention, à une volonté de faire signe (il se fait "par le commandement de l'âme»), c'est parce qu'il est le seul aussi, selon toute vraisemblance, à représenter mimétiquement le mouvement que l'âme "souffre intérieurement» (le seul, donc, dont le statut de signe et la lisibilité soient garantis par une ressemblance qui saute aux yeux). De l'autre, "tout le reste» - soit divers effets corporels purement involontaires, dont Cureau suppose qu'ils résultent mécaniquement de la contraction des muscles (dont ils sont "une suite nécessaire») et que l'on pourrait désigner comme effets secondaires: ils ne procèdent d'aucune intention de faire signe (en quoi ils sont coupés de la finalité sociale globalement assignée au rire), et s'ils peuvent être déchiffrés comme indices (au sens de Peirce) de la "Passion du Ris», ces "caractères» d'un autre genre n'en peignent pas «l'image et la figure». Or, "tout le reste» est beaucoup plus qu'un simple reste; loin de se trouver confinés à la marge, ces signes involontairement prodigués et privés 40. M. Cureau de la Chambre, Les Charactères des Passions, op. cit., p. 206-207 (je souligne). 
de valeur iconique tendent à constituer l'essentiel de l'ample description des "principaux traits qui ont accoutumé de former le Ris véhément", description qui mérite à cet égard d'être reproduite:

Car si vous considérez le Visage, le front s'étend, les sourcils s'abaissent, les paupières se resserrent au coin des yeux, et toute la peau qui les environne se rend inégale et se couvre de rides. Les yeux s'apetissent et se ferment à demi, ils deviennent brillants et humides: et ceux-là même de qui la douleur n'a jamais pu tirer des larmes sont alors contraints de pleurer. Le nez se fronce et se rend aigu, les lèvres se retirent et s'allongent, les dents se découvrent, les joues s'élèvent et se rendent plus fermes, et quelquefois leur milieu se creuse doucement et forme ces agréables fossettes où les Poètes ont logé le Ris et les grâces. La bouche qui est contrainte de s'ouvrir fait voir la langue qui se trémousse et qui se tient suspendue; et la voix qui en sort n'est rien qu'un son éclatant et entrecoupé que l'on ne saurait arrêter, et qui ne finit qu'avec la perte de l'haleine. Le col s'enfle et se raccourcit, toutes les veines sont grosses et tendues, un certain éclat agréable se répand sur tout le visage, et quelque pâle et sévère qu'il soit, il faut qu'il rougisse et qu'il paraisse content.

Mais tout cela n'est rien à comparaison de ce qui se fait dans les autres parties: la poitrine s'agite si impétueusement et par des secousses si promptement redoublées que l'on a de la peine à respirer, que l'on perd l'usage de la parole, et qu'il est impossible d'avaler quoi que ce soit. Une douleur si pressante s'élève dans les flancs, qu'il semble que les entrailles se déchirent et qu'elles se vont ouvrir; et dans cette violence on voit tout le corps qui se plie, se tord et se ramasse; les mains se jettent sur les côtes et les pressent vivement; la fureur monte au visage, la voix se perd en sanglots et l'haleine en soupirs étouffés. Quelquefois cette agitation va à tel excès, qu'elle produit le même effet que les médicaments, qu'elle chasse les os de leurs jointures, qu'elle cause des syncopes, et qu'enfin elle donne la mort. La tête et les bras souffrent les mêmes secousses que la poitrine et les flancs, mais parmi ces mouvements vous voyez qu'ils se jettent çà et là avec précipitation et désordre, et qu'après ils se laissent aller d'un côté et d'autre, comme s'ils avaient perdu toute leur vigueur, les mains deviennent lâches, les jambes ne se peuvent soutenir et le corps est contraint de tomber. ${ }^{4 r}$

Cette ample description (qui donne la mesure de la prolixité de Cureau et semble gagnée par un effet d'emballement comparable à celui dont elle rend compte!) fait assez voir que le rire, s'il est "principalement destiné pour la conversation ", contrevient cependant, dans sa forme marquée ("véhémente»), aux codes qui la régissent à l'âge classique: que les effets du Ris puissent aller non seulement "jusqu'à des contorsions qui défgurent le visage», comme le déplorera le père Le Moyne ${ }^{42}$, mais encore jusqu'à l'étouffement, jusqu'au spasme, jusqu'à l'évanouissement, jusqu'à la mort même..., voilà qui suffit sans doute à le rendre suspect aux yeux des législateurs de la civilité et qui fait bien comprendre pourquoi «le rire n'a pas toujours très bonne réputation au XVII ${ }^{e}$

4I. Ibid., p. 179-180.

42. Les Femmes, la modestie et la bienséance chrétienne, Paris, I667, p. I87 (je souligne). 
siècle43». Mais au-delà de la démesure (ou de la véhémence) qui suffit en ellemême à le disqualifier, l'inadéquation du rire à la "vie civile» semble tenir au double soupçon d'insignifiance et d'inhumanité que la description de Cureau fait incidemment peser sur la mécanique des éclats, dont elle donne à voir, non sans une certaine fascination, l'inquiétante étrangeté: que dit le rire? sinon le reflux toujours possible de tout cela qui dans l'homme n'est pas l'homme, mais animal ou machine, animal et machine.

Il est remarquable, aussi bien, que le médecin ne parvient pas à faire entrer tous les effets du rire dans le cadre de l'explication finaliste qu'il se donne pourtant pour horizon, et qu'il échoue à les rapporter à la fonction figurative qu'il assigne pourtant aux "Caractères corporels» : on ne voit pas ce que tout cela veut dire, parce que tout cela ne ressemble à rien... Rire sonore, le "Ris véhément" fait entendre une "voix qui se perd en sanglots», "rien qu'un son éclatant et entrecoupé», un "Mugissement entrecoupé» - un langage inarticulé, voisin du cri de l'animal, un entre-deux entre la parole et le silence 44 , et dont l'insertion dans le champ de la conversation ne laisse pas d'être problématique. Rire spasmodique et mortifere, il n'est pas sans parenté avec les désordres de l'amour: comme le notait Laurent Joubert au siècle précédent, le rire est avec l'émotion sexuelle ce qui échappe à la volonté raisonnable. De fait, à la défaillance du modèle iconique, qui permet idéalement d'attribuer aux effets corporels une fonction de représentation, s'ajoute la défaillance du modèle autocratique qui les subordonne en principe à la volonté de l'âme: c'est peu dire en effet que le corps-automate prend ici les commandes, échappe au "commandement de l'âme" - à un processus de déclenchement volontaire du rire - et s'emballe, sans que la volonté y ait sa part. Le "Ris véhément", à raison même de sa véhémence, ouvre la voie à ce dessaisissement des prérogatives de l'âme-autocrate qui affecte et reflue jusqu'à ce «mouvement des muscles» qui relève pourtant de son "commandement":

C'est donc une chose certaine que le mouvement des muscles qui forme le Ris est une action volontaire qui se fait par le commandement de l'âme et non point par nécessité, comme se font les larmes, la sueur, l'éclat et la rougeur du visage [" effets secondaires» de la Passion du Ris] : c'est pourquoi on le peut empêcher et retenir au commencement, lorsque les humeurs et les esprits ne sont pas encore fort

43. J. Morel, «Rire au XVII siècle», Quaderni del seicento francese, nº Io, Paris, Nizet, I992, p. 27. On sait que le goût classique marque sa préférence pour les formes atténuées, voire quasi désincarnées, du rire, à l'instar de Donneau de Visé qui, dans sa Lettre écrite sur la comédie du "Misanthrope», loue la grande comédie de ce qu'elle fait "rire dans l'âme».

44. Sur cet aspect et ses implications anthropologiques, voir D. Bertrand, «Bruit et silence: la voix rieuse au XVII e siècle, ses enjeux scientifiques, sémiotiques et disciplinaires", Littératures classiques, $\mathrm{n}^{\circ} \mathrm{I} 2$, janvier I990, p. IOI-II5. 
ébranlés; et de là vient que bien souvent, tenant la bouche fermée, l'haleine et la voix qui sont contraintes de passer par les narines causent ce Mugissement entrecoupé qui se remarque dans le Ris.45

Que la passion du Ris prenne l'âme par surprise, ce dispositif de maîtrise est alors neutralisé:

Dans cette précipitation, l'âme n'a pas le temps de discerner ce qui se fait, et les parties sont plus tôt émues qu'elles ne s'en est avisée. Et pour lors elle n'est pas capable de retenir le branle qu'elle s'est donné; les esprits et les humeurs en ayant reçu l'impression, dont l'impétuosité ne se peut arrêter si promptement. Et de là vient qu'il est très difficile d'empêcher le Ris quand il est vébément, quoi que ce soit une action volontaire; tout de même qu'il arrive dans les autres passions où l'âme souffre la même violence que celui qui court dans un précipice: car bien que ce soit lui qui se soit donné le mouvement, il n'est plus en son pouvoir de l'arrêter; il faut qu'il s'abandonne au branle quỉl a pris, et au penchant où il s'est précipité. 46

On mesure que les effets de vitesse acquise qui régissent la mécanique des éclats prennent ici le pas sur on ne sait (plus) quelle intention de faire signe (signe de quoi, on ne le sait pas davantage). Et l'on devine, du coup, l'affleurement d'un autre modèle, celui de l'automate, dont D. Bertrand a montré qu'il informe fréquemment, dans la première moitié du siècle, les représentations du corps rieur ${ }^{47}$, et qui joue chez Descartes le rôle essentiel que l'on sait.

Aussi bien, s'il est un enseignement que l'on peut tirer de cette lecture des "Caractères du Ris», c'est que l'imposant appareil logico-rhétorique déployé par Cureau (appareil dont le traité cartésien prendra, comme on le sait, le contre-pied) dissimule finalement assez mal de très remarquables distorsions théoriques: qu'un discours puisse en cacher un autre, c'est une vérité générale qui se révèle peut-être, chez un auteur de transition comme celui des Caractères des Passions, un peu plus vraie qu'ailleurs... De fait, s'il est certain que la théorie du rire qui s'y trouve exposée est très éloignée d'exclure «la ressemblance comme expérience première et fondamentale du savoir $4^{8}$ ", il n'en est pas moins vrai qu’à bien des égards elle esquisse le chemin qui va mener à Descartes - quand bien même ce chemin y prend provisoirement la forme d'une impasse!

Pour esquisser, en guise d'épilogue, une brève comparaison, on insistera

45. M. Cureau de la Chambre, Les Charactères des Passions, op. cit., p. 2 I4 (je souligne).

46. Ibid., p. 202 (je souligne).

47. «L'image de l'automate dans les représentations du rire et du ridicule au XVII siècle», Lectures, $\mathrm{n}^{\circ}$ I9 ("Automi»), 1986 (Bari, Edizioni dal Sud), p. I9-29.

48. M. Foucault, Les Mots et les choses..., op. cit., p. 66. 
sur l'importance dévolue par chacun des deux auteurs, du double point de vue de la théorie des passions et de la rhétorique de la conversation, à la question du rire-importance dont on a vu qu'elle se manifeste chez Cureau par le biais d'un artifice taxinomique qui lui permet, en élevant le Ris à la dignité de passion, de lui consacrer un chapitre tout entier (et davantage d'ailleurs). Il est permis de penser que le cas du "Ris véhément", cas-limite dont Cureau choisit pourtant de faire son principal objet d'étude, revêt une signification comparable à celle que Descartes va conférer, dans Les Passions de l'âme, au cas du rire-réflexe, dont il a trouvé l'exemple chez Vivès et dont il a fait l'expérience par lui-même:

[...] Et généralement tout ce qui peut enfler subitement le poumon en cette façon, cause l'action extérieure du ris; excepté lorsque la tristesse la change en celle des gémissements et des cris qui accompagnent les larmes. À propos de quoi Vivès écrit de soi-même que, lorsqu'il avait été longtemps sans manger, les premiers morceaux qu'il mettait en sa bouche l'obligeaient à rire: ce qui pouvait venir de ce que son poumon, vide de sang par faute de nourriture, était promptement enflé par le premier suc qui passait de son estomac vers le cœur, et que la seule imagination de manger y pouvait conduire, avant même que celui des viandes qu'il mangeait y fût parvenu. 49

Dans cet exemple, en effet, la machine corporelle est close sur elle-même et le rire (n')est (qu')un pur automatisme physique: c'est là le rire irréfléchi et involontaire de l'homme-machine, que la description du "Ris véhément» chez Cureau préfigure à bien des égards. Or, au-delà même de ce cas-limite, l'importance accordée par Descartes aux remarques sur le Ris tient au fait que le phénomène, comme le signale J.-M. Monnoyer, semble «marquer plus nettement que tout autre l'automatisme physique séparé de sa cause réelle ${ }^{50}$ ", illustrant exemplairement la dissociation de la cause psychique et du mécanisme corporel dans la théorie cartésienne des passions. On sait que, pour autant, l'hypothèse de la glande pinéale - hypothèse centrale du traité cartésien - permet d'ouvrir le système clos de la machine corporelle à l'intervention de l'âme et, partant, rouvre la voie à une maîtrise raisonnée du rire en contexte d'interaction sociale, ce qui revient à renouer le fil - mais sur des bases nouvelles - entre rire et conversation. Un fil que l'on a vu se défaire, avec celui de la ressemblance, quelque part entre les lignes, au milieu du texte de Cureau.

49. Descartes, Les Passions de l'âme (éd. G. Rodis-Lewis), op.cit., art. I27.

50. Voir l'édition des Passions de l'âme procurée par J. -M. Monnoyer, Paris, Gallimard (Tel), I988, p. 283-284, n. 23. 